\title{
Machine Learning based Identification and Classification of Field-Operation caused Solar Panel Failures observed in Electroluminescence Images
}

\author{
Stefan Bordihn, Andreas Fladung, Jan Schlipf and Marc Köntges
}

\begin{abstract}
Failure or degradation effects lead to power losses in solar panels during their field operation and are identified commonly by electroluminescence (EL) imaging. Some failures like potential induced degradation (PID) and light and enhanced temperature induced degradation (LeTID) require an identification of the EL pattern over the entire solar panel. As the manual process of analysing patterns is prone to error, we seek for an automatic detection of these failure types. We predict automatically the failure types PID and LeTID by adopting the principle component analysis (PCA) method in combination with a knearest neighbour $(\mathrm{kNN})$ classifier. We increase the explained variance of the PCA by $4 \%$ abs using a Gaussian blurring pre-processing step and gain insights into the basic mechanism of the machine learning algorithm by analysing schematic EL images. The $\mathrm{kNN}$-classifier predicts the failure classes in the same way as the expert. Finally, we work with a larger test data set of 40 similar images to mimic a field-typical user case and meet again the expert classification.
\end{abstract}

Index Terms-Electroluminescence Imaging, Machine Learning, Silicon Solar Panels, Field Operation Failures.

\section{INTRODUCTION}

M ost failures that lead to a reduction of the output power of solar panels during their field operation can be identified with the electroluminescence (EL) imaging technique [1], [2]. The expression in the EL image of some failure types is restricted to the size of the individual solar cells, e.g. series resistance losses due to detached metallization, cracked wafers, decoupled solar cell areas and many more. In contrast, failures like potential induced degradation (PID) [3] and light and enhanced temperature induced degradation (LeTID) [4] are separated by analysing the specific EL-signal pattern over the entire solar panel dimension or even a string of solar panels. However, both effects look quite similar, especially at the beginning of the degradation process. Moreover, the strength of the degradation and the resulting pattern depend on the mounted orientation (landscape or portrait) in the system and the applied stress by the environment, e.g. seaside location, and climate.

This work was funded by the German State of Lower Saxony and the ZIM program of the Federal Ministry for Economic Affairs and Energy within the research project AERIALPVREVIEW (Project number ZF4523002LT9).

S. Bordihn and M. Köntges are with the Institute for Solar Energy Research Hamelin/Emmerthal (ISFH), 31860 Emmerthal, Germany (email: bordihn@isfh.de; koentges@isfh.de).
Hence, a manual classification by an expert can be prone to error. Therefore, many studies deal with the development of an automatic separation of failure types on solar cell level, e.g. Ref. [5] and references therein, or extracting solar cells from an EL image of a solar panel, e.g. Ref. [6], [7]. To our knowledge, no study considers the entire solar panel EL image during the analysis to identify PID or LeTID defects.

In this study, we adapt the principle component analysis (PCA) method to distinguish between two of the most important outdoor failure types: PID and LeTID. This is important because each failure type results in different mitigation strategies for affected PV systems. The failure types are identified on basis of EL images measured in field tests. We select the PCA because there is no need of pre-labelling the investigated EL images by the expert. We use the cumulative explained variance as a figure of merit to evaluate the quality of the failure separation. In a second step, we add schematic EL images to the training data set to highlight the main features of the PID defect and to gain a basic understanding of the way the PCA separates between typical images. A kNN-classifier predicts the failure class on basis of the PCA transformed data. Finally, a larger test data set of 40 EL images taken from one field test is used to simulate a typical user case. Fig. 1 depicts the data flow in form of consecutive steps as an orientation to the reader. In Sec. IV the training data set is extended by schematic EL images as aforementioned and in Sec. V the test data set is replaced by a larger data set of similar EL images to mimic a typical use case.

\section{DATA AND METHOD}

\section{A. Sample set}

The training data set consists of 54 EL images separated by experts into the classes: (A) Reference (mostly non-affected by degradation), (B) LeTID and (C) PID. This pre-separation into classes serves as ground-truth and serves as orientation to the reader whether the PCA separates the data set like the expert. We would like to stress out that there is no strict definition whether a solar panel suffers from PID or LeTID, especially, when the defect characteristics are at the beginning of their

A. Fladung and J. Schlipf are with Aerial PV Inspection GmbH, 52064 Aachen, Germany. 
evolution. In this case, the typical distribution of optical and electrical variation of the solar cells can lead to similar EL patterns. From a physical point of view, all crystalline Si solar panels suffer to some extend from PID and LeTID but it is not visible/ measurable when other effects dominate the EL image. Moreover, in rare cases the solar panel manufacturer intentionally adds a specific variation, i.e. selection of solar cells, which can lead to a wrong interpretation by the expert, see e.g. cell mixing whitepaper of Ref. [8]. In this study, we have therefore considered more or less typical images for each type of defect, with the aim of realising a proof of concept for the analysing procedure presented.

Fig. 2 gives a graphical summary of all 18 EL images per failure class. The images are not consistent in their underlying solar cell technology, wafer size and shape to increase the variability of the data. Solar panels with solar cells based on multi- and mono-crystalline $\mathrm{Si}$ materials are included to increase the variability, too. The solar panel mounting in the field is either landscape or portrait orientation. During the data processing step (Sec. II-C) the corresponding bottom edge of the solar panel (and EL image) is either at the bottom or right side. This consistency is of major importance due to orientation dependence of the PID failure and therefore to generate a clear training data set for the machine learning algorithm. A high variability of the data set is desired as well to generate a method that is as general as possible. Additionally we include EL images from literature to be to some extent independent of systematic errors of our own EL measurement system. In the LeTID class (B), the image with the ID 15 is adapted from Ref. [9] and 16 from Ref. [4]. In the PID class (C), the image with the ID 2 is adopted from Ref. [10], ID 4 from Ref. [11] and ID 6 from Ref. [12]. The EL images are measured with the same setup within one type of solar panel and failure class except for the literature images. For the latter no measurement and image scaling details are known which is a meaningful uncertainty that increases further the variability of the data set. We use image acquisition parameters adapted to the solar panel type and failure type. This is necessary as the EL signal obtained from the solar panel varies significantly, e.g. it is dependent on multior mono-crystalline solar cells.

\section{B. Principle Component Analysis}

The principle component analysis (PCA) is an unsupervised machine learning algorithm [13]. It reduces higher dimensional data to lower dimensional data, meaning it identifies the main trends that define a variation of a data set. The number of trends is called components, and it depends on the target of the analysis which number of components is sufficient to accurately describe the data set. In principle, a higher number of components describes the training data set more accurately but the chance of overfitting is also higher. The latter is undesired to generate an algorithm that is as general as possible, i.e. which is able to perform well on unknown data sets as well. The variance that is explained by each of the selected components gives a measure to decide for a specific number of components.

\section{Nearest Neighbour Classification}

The near neighbour algorithm is commonly adopted for classification, i.e. prediction of a failure class [14]. In principle, the kNN method is a distance analysing algorithm. An unclassified data point is assigned to the main class of its closest neighbour. By main class we refer to the class that most of the neighbour belongs to. The user needs to specify the number of neighbours $k$ and accordingly the method is referred to as kNN classifier. In our case, we aim to predict the PCA transformed data of an unknown EL image to one of the three pre-defined failure classes, so $k=3$ in our case. The prediction occurs with a specific certainty that helps the user to judge about the quality, i.e. accuracy, of the prediction.

\section{Data processing}

We use Python as programming language. We import the EL images as grayscale image using the opencv framework [15]. We rescale all images to $x$-dimension of 120 pixel and $y$ dimension of 240 pixel to reduce the computational effort. Hence, we analyse 28,800 feature dimensions. The reduction of the image dimensions did not affect the achieved results presented in this work. We achieved the same results when the images are scaled to $1,200 \times 2,400$ pixel, i.e. larger by a factor of 100. The images are transformed into a one-dimensional numpy [16] array that represents the EL signal intensity and its corresponding $x$ - and $y$-positions but flattened to a onedimensional row. The sklearn [17] package provides the PCA analysis tools. The resulting principle components (PC) are numbered through $(\mathrm{PC} 1, \mathrm{PC} 2, \ldots)$ by their importance for representing the diversity of the underlying data set. In a second step, we optimize the separation quality of the PCA by an image pre-processing step but keeping the number of PCs constant. The pre-processing step is a Gaussian blurring process. The intention is to reduce the effect of local information in the EL images, e.g. busbar shading, finger interruptions, wafer material marks, which is not relevant for the separation between failures that are identified by observing the entire solar panel area, see Fig. 3. The kernel of the blurring is varied between $1 / 24$ to $1 / 4+1$ as a fraction of the $y$-dimension of the EL image. Therefore, the kernel values are odd numbers as it is technically required.

\section{EVALUATION OF FAILURE IDENTIFICATION}

\section{A. PCA Seperation of Failure Classes}

We evaluate the impact of the convolution kernel size on the PCA separation on basis of the cumulative explained variance, see Fig. 4-a. The convolution kernel with the size of $1 / 24$ already increased the cumulative explained variance by $3.9 \%$ abs and the kernel with the size $1 / 4+1$ of the $y$-dimension of the EL image increased the cumulative explained variance by $5.8 \%$ abs. For all following analysis, we keep the blurring kernel of $1 / 24$ to reduce the information given by the spacing of the solar cells or busbars and to keep more details of larger image structures.

The optimal number of principle components (PCs) can be identified using the Scree plot. The optimal number of PCs describe most of the original data set but not too specific. The Scree plot shows the explained variance over the number of 
principle components (Fig. 4-b). The common way of selecting the optimal number of PCs is to use the value given at the indicated bending point that is in our case two. Of course, the PC1 provides the main differentiation with a cumulative explained variance of $74.2 \%$ while PC2 accounts for additional $6.5 \%$. Hence, the first two components describe about $81 \%$ of the variance of the original data.

Fig. 4-c depicts PC2 as a function of PC1. The number close to the data point helps to correlate each data point to its original EL image (Fig. 2). The obtained values of the solar panels of each failure group are located in distinguishable clusters although the points of the PID class scatter more than the other classes. The clustering separates the failure classes in the same way as given by the ground truth, i.e. the expert classification. Hence, the PCA works in the desired way and can be used as a data processing step to facilitate a classification step.

\section{B. Understanding of PCA Separation}

We added schematic EL images to the training data set to gain a basic understanding on which features the machine learning algorithm separates the EL images. Fig. 5 replots the results shown in Fig. 4-c and, additionally, as red dots the results obtained for the schematic EL images shown on the right-hand side of Fig. 5. From left to right the homogenous brightness of the EL images ID 1-3 leads to a certain separation of the PCA transformed data. Hence, we assume that the overall brightness has a major impact on the PCA. The specific pattern of the schematic EL images with the ID 4-6 gives a separation of the PC data from bottom to top in Fig. 5. The achieved PCA results of those images lie close to the data points of real EL images that show the same features, see for comparison Fig. 2. Hence, the second major impact on the PCA is triggered by a strong contrast variation over solar cell rows or columns in the solar panel.

\section{C. kNN Failure Classification}

We adopt a $\mathrm{kNN}$ classifier to predict the failure class of 18 test EL images on basis of the PCA transformed data (see Fig. 2). Fig. 6 shows the resulting confusion matrix with respect to the reference class. The chosen test images consider some new solar panel technologies like the half-cell design that is not used during training. Nevertheless, the class of the solar panel with the half-cell technology is predicted correctly, see true positive (TP) result in Fig. 6-c. Also for the LeTID class, we added images that have not been used during training in the sense that the camera settings and image corrections are unknown. Two of those images result in a wrong classification, i.e. false positive (FP) prediction. This is a result of a very small manifestation of the LeTID failure, even for the expert the difference between the FP image shown in Fig. 6-c is hard to separate from the reference images given in the training data set. Moreover, we also added three images with a proven PID failure from a field test, which had been provided by Ref. [18], to the test image set to check again whether our method works on images with unknown camera settings and applied image correction. The failure class of one of those three images is predicted as true negative $(\mathrm{TN})$ with a wrong prediction, i.e. it was predicted as LeTID failure (Fig. 6-c). This is possibly caused by the distortion of the image.

\section{CASE Study}

For an application case, we consider 40 EL images from a field test that are very similar in their appearance. This data is used as test data set in the flow chart given in Fig. 7. The PCA again transforms the main features of the EL images into a three dimensional data set. Fig. 7 shows the PCA results together with those described in Sec. III-A. In contrast to the procedure given in Fig. 1 the kNN classifier analyses the mean of PC1 which is 4,165 and the mean of PC2 which is $-1,311$ instead of every individual value. The reason for this is that under field circumstances multiple defect types can harm the solar panel performance. For example, PID and LeTID are to some extent present in every solar panel in the field. Therefore, the expert commonly investigates a group of solar panels and reports a main defect type to the system owner. The presented example leads to the kNN predication of LeTID as the most dominant failure type.

\section{CONCLUSIONS}

In this work, we adapted the unsupervised machine-learning algorithm PCA and combined it with a kNN classifier to predict whether unknown EL images of solar panels suffer from PID or LeTID that are two of the most dominant failure types in today's field-installed solar panels. For the PCA step, we identified that the first three components separate the failure classes in sufficient manner. We increased the separation quality by $6 \%$ abs using a Gaussian blurring pre-processing step to the EL images. We present a proof of concept by showing a successful prediction of the failure class by the combined PCAkNN algorithm. We achieve, except for one example, a prediction probability of 1 . This holds true also for solar panels based on a technology and measurement settings not considered in the training data set. Furthermore, we gained more insights into the separation mechanism of the PCA by using schematic EL images. Finally, on basis of a mimicked case study we propose that analysing the mean value of the PCA transformed data can serve as a meaningful basis for the following $\mathrm{kNN}$ classification of a field-typical data set where a mixture of failure types are present.

\section{REFERENCES}

[1] T. Potthoff, K. Bothe, U. Eitner, D. Hinken and M. Köntges, "Detection of the voltage distribution in photovoltaic modules by electroluminescence imaging", Prog. Photovoltaics Res. Appl. 18, 2 (2010) pp. 100-106.0.

[2] IEC TS 60904-13:2018 Photovoltaic devices - Part 13: Electroluminescence of photovoltaic modules, "Electroluminescence of photovoltaic modules." IEC, 2018.

[3] S. Pingel, O. Frank, M. Winkler, S. Daryan, T. Geipel, H. Hoehne and J. Berghold, "Potential induced degradation of solar cells and panels", in Proc. of 35th IEEE Photovoltaic Specialists Conference, Honolulu, Hawaii, USA (2010).

[4] F. Kersten, P. Engelhart, H.-C. Ploigt, A. Stekolnikov, T. Lindner, F. Stenzel, M. Bartzsch, A. Szpeth, K. Petter, J. Heitmann, and J. W. Müller, "Degradation of multicrystalline silicon solar cells and modules 
after illumination at elevated temperature", Sol. Energy Mater. Sol. Cells 142, 83 (2015).

[5] S. Deitsch, V. Christlein, S. Berger and C. Buerhop-Lutz, "Automatic Classification of Defective Photovoltaic Module Cells in Electroluminescence Images“, Solar Energy 185 (2018).

[6] H. R. Parikh, Y. Buratti, S. Spataru, F. Villebro, G. A. Dos Reis Benatto, P. B. Poulsen, S. Wendlandt, T. Kerekes, D. Sera and Z. Hameiri, "Solar Cell Cracks and Finger Failure Detection Using Statistical Parameters of Electroluminescence Images and Machine Learning", Appl. Sci. 10 (2020).

[7] S. Deitsch, C. Buerhop-Lutz, A. Maier, F. Gallwitz, C. Riess, "Segmentation of Photovoltaic Module Cells in Electroluminescence Images", arXiv:1806.06530 (2018).

[8] Hanwha Q Cells' white paper: cell mixing, https://www.qcells.us/na/main/resources/download/whitepaper.html (last access 2021/23/05)

[9] F. Kersten et al., "Feldrelevante Degradationseffekte von multikristallinen Siliziumsolarzellen \&-modulen“, 12. Workshop on Photovoltaik-Modultechnik, Cologne, Germany (2015).

[10] Report IEA-PVPS T13-10:2018, "Review on Infrared and Electroluminescence Imaging for PV Field Applications", ISBN 978-3906042-53-4.

[11] K. Shahzad et al. "Addressing potential-induced degradation of fieldinstalled PV modules by reducing surface conductivity", Proceedings of New Concepts in Solar and Thermal Radiation Conversion and Reliability (2018), San Diego, California, USA.

[12] Luo et al., "Potential-induced degradation in photovoltaic modules: a critical review", Energy Environ. Sci. 10 (2017).

[13] G. H. Dunteman, Principal Components Analysis, Sage Publication 1989 ISBN: 9780803931046.

[14] J. M. Keller, M. R. Gray, and J. A. Givens, “A Fuzzy K-Nearest Neighbor Algorithm", IEEE Trans. Systems, Man, and Cybernetic 15, 4 (1985) pp. 580-586.

[15] https://opencv.org/ (last access 2021/03/05)

[16] https://numpy.org/ (last access 2021/03/05)

[17] https://scikit-learn.org/ (last access 2021/03/05)

[18] Courtesy of Ingenieurbüro Fleiss, Springe-Eldagsen Germany.

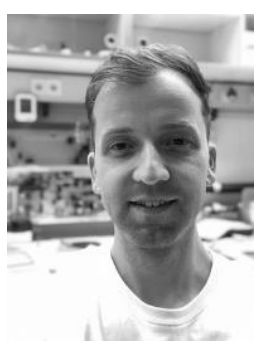

Stefan Bordihn received his B.Sc. ad M.Sc. from the University of Applied Science Berlin in Renewable Energy Systems in 2007 and 2010, respectively. He has done his Ph.D. at Hanwha Q Cells and the Eindhoven University of Technology in the field of plasma-based thin film processing for high-efficiency solar cells (2014). He worked as expert and project leader at Hanwha Q Cells until he joined the Institute for Solar Energy Research (ISFH) in 2018 where he develops machine learning algorithms for the application in the field of photovoltaic research.

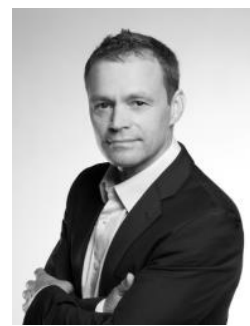

Andreas Fladung studied electrical science at the University of Applied Science in Aachen after he finished his professional education as electrician. In 2005 he founded the Fladung Solartechnik GmbH. He is one of the world leading experts in characterization of large PV systems using thermography and electroluminescence imaging techniques. In 2017, he founded Aerial PV Inspection $\mathrm{GmbH}$ where he is responsible for the project development and acquisition as executive officer.

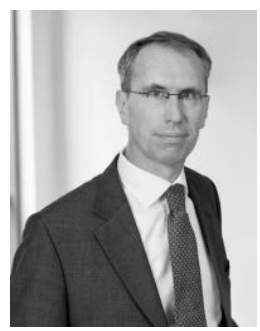

Jan Schlipf received his $\mathrm{Ph} . \mathrm{D}$. on magnetism in many-particle physics from the University of Augsburg in 1998. He worked several years in the automotive industry with a focus on automatic transmission controls. From 2003-2015 he was part of the company management of the Gesellschaft für Industrieforschung mbH. In 2017 he founded Aerial PV Inspection $\mathrm{GmbH}$ and beside his role as managing director, he develops algorithms to analyse data from field measurements of large PV systems.

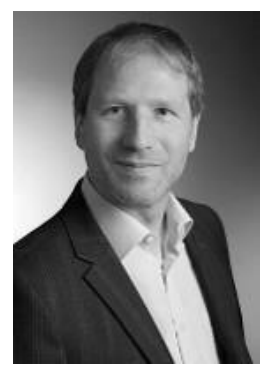

Marc Köntges received the Ph.D. degree in physics, on the characterization of CuInGaSe2and CdTe thin film solar cells, from the University of Oldenburg, Oldenburg, Germany, in 2002. Since 2002, he has been leading the thin-film technology group with the Institute for Solar Energy Research Hamelin (ISFH), Emmerthal, Germany. In 2005, he went on to lead the photovoltaic (PV) module and interconnection group with ISFH. He currently develops characterization and production methods for PV modules. 
1. Input

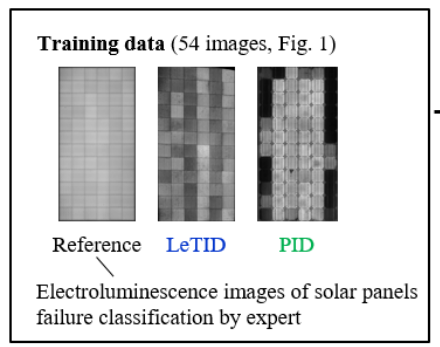

2. Pre-Processing

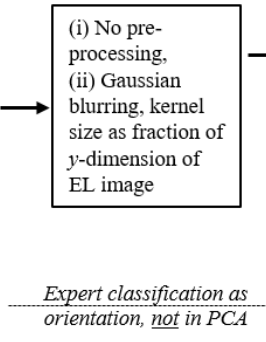

3. PCA

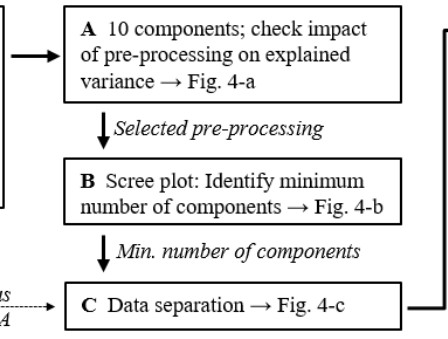

4. kNN Classifier

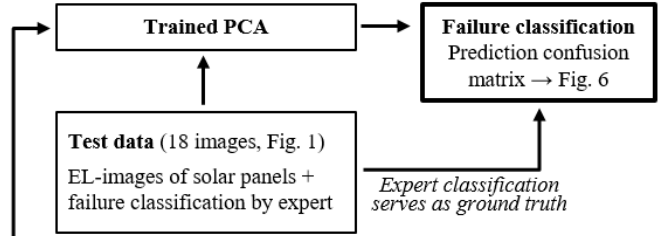

Fig. 1: Schematic overview of applied data flow. For the result given in Fig. 5 the input data is extended by artificial EL images. For the results shown in Fig. 7 the test data set is replaced by a larger set of field-measured EL images.

\section{TRAINING DATA SET}

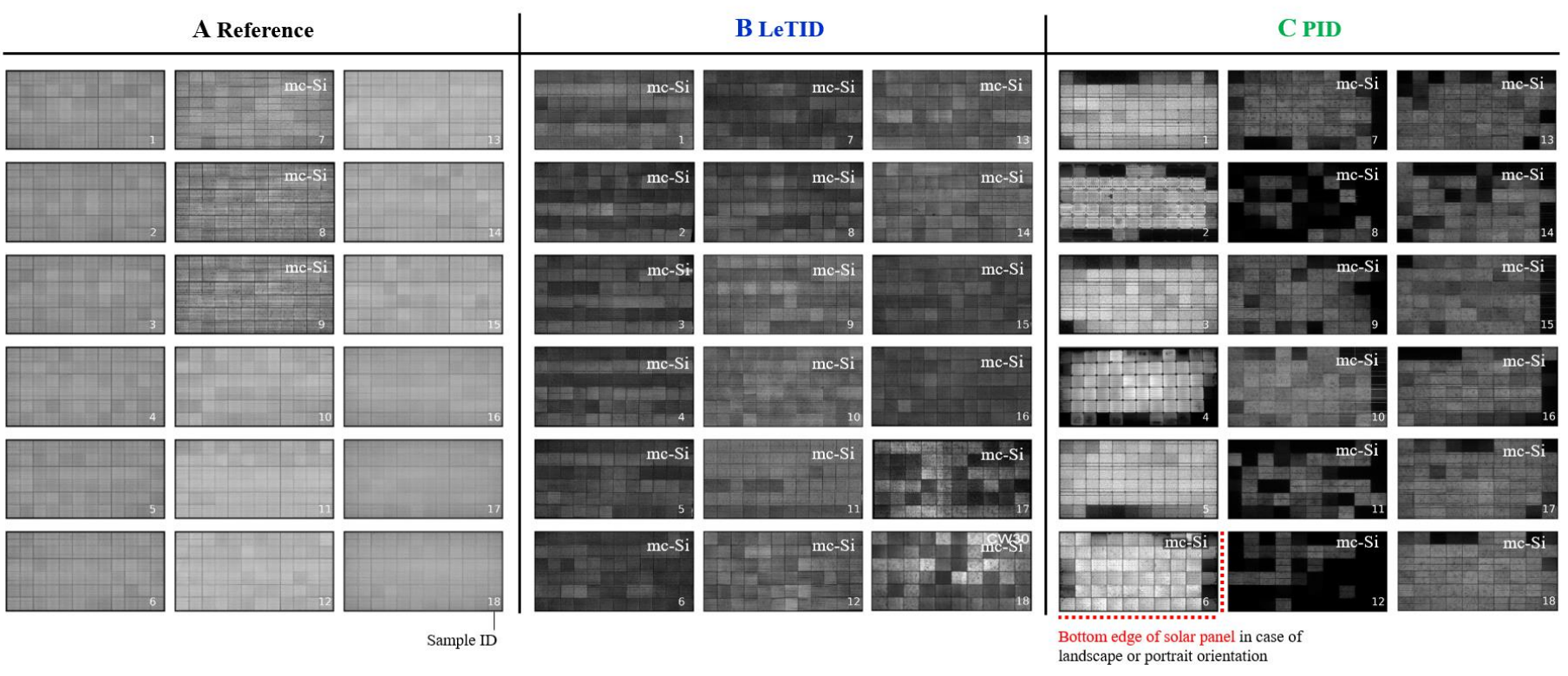

\section{TEST DATA SET}
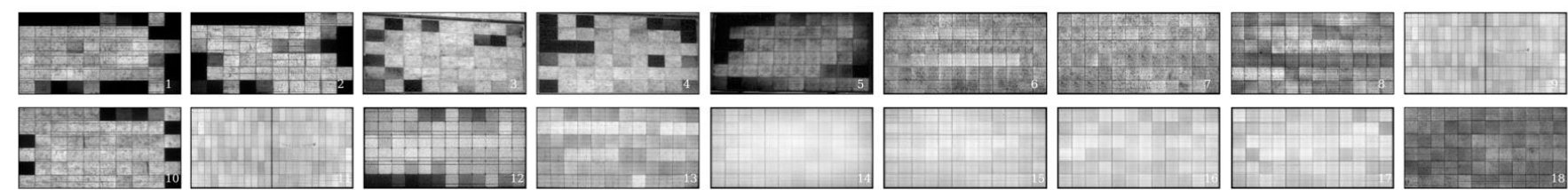

Fig. 2: Top: Training data set of 54 electroluminescence images separated into classes by expert choice (ground-truth). Class A serves as a reference with no obvious failure. Class B gives examples of the light and elevated temperature induced degradation (LeTID) and class C shows examples of the potential induced degradation (PID). Class B and C includes images taken from literature: in B: \#17 Ref. [9] and 18 Ref. [4] and in C image \#2 Ref. [10], \#4 Ref. [11] and \#6 Ref. [12]. Bottom: Test data set contains 18 images, including half-cell technology based solar panels. 
Gaussian blurred Images with kernel as fraction of $y$-dimension of EL image

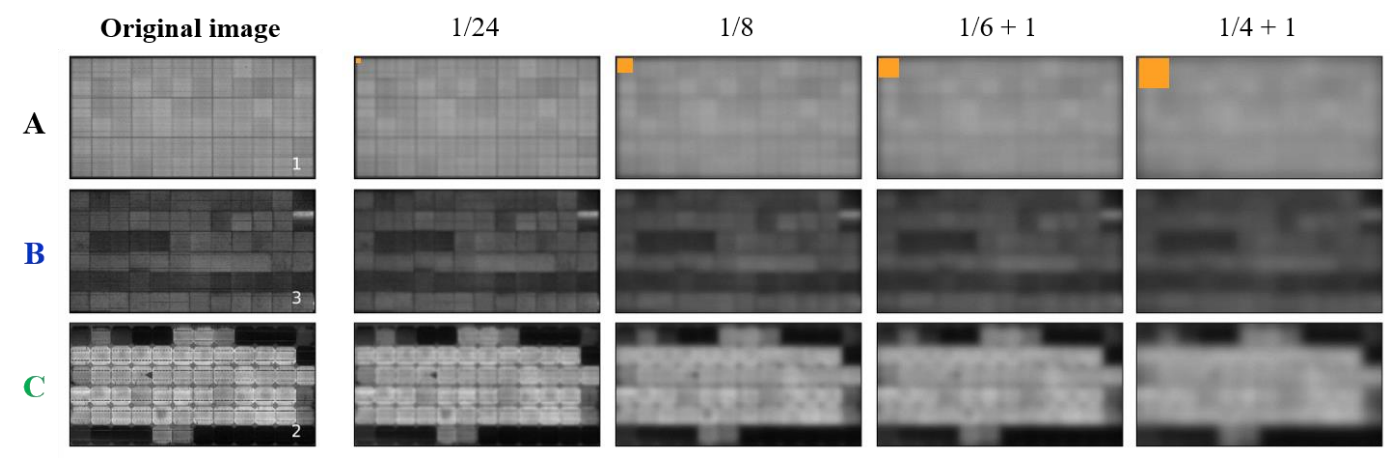

Fig. 3: One example EL image of each class of Fig. 2 and its evolution due to the Gaussian blurring that is adapted as pre-processing step in Sec. III-B. The size of the blurring kernel is varied as a portion of the y-dimension of the EL image.
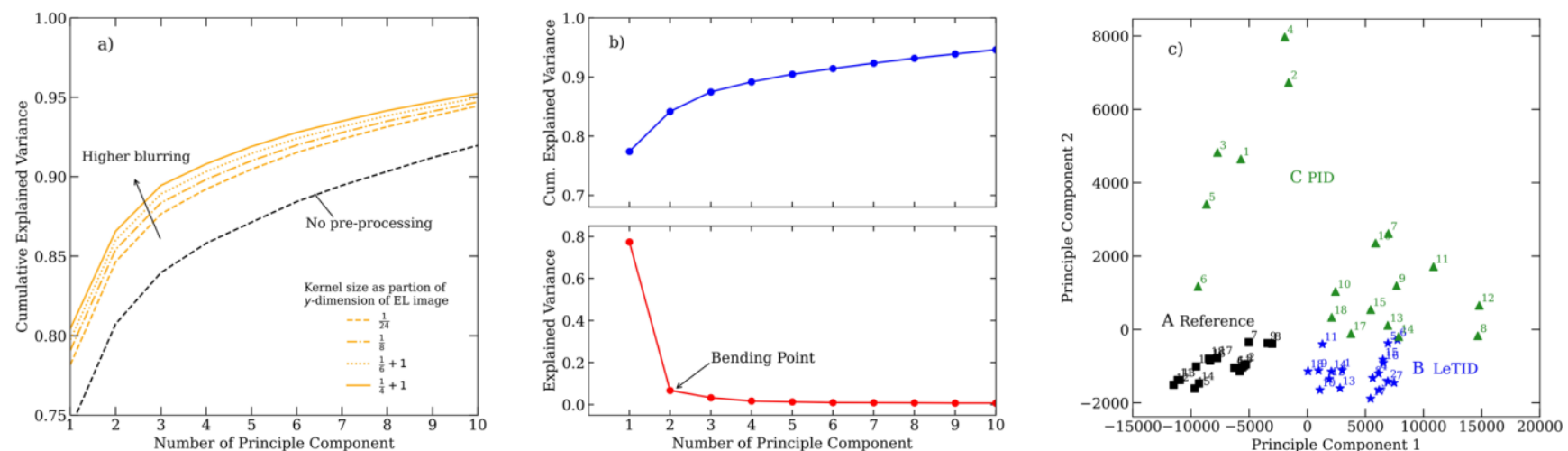

Fig. 4: (a) Cumulative explained variance as a function of the number of principle components of the PCA. Data obtained with the original EL images in black and results achieved by using blurred EL images given in orange lines. (b) Cumulative explained variance and explained variance as a function of the number of principle components. The bending point indicates the number of components needed to describe the original data set. (c) Dependence of PC2 as a function of PC1 obtained after PCA. The colors indicate the failure classes of the ground-truth.
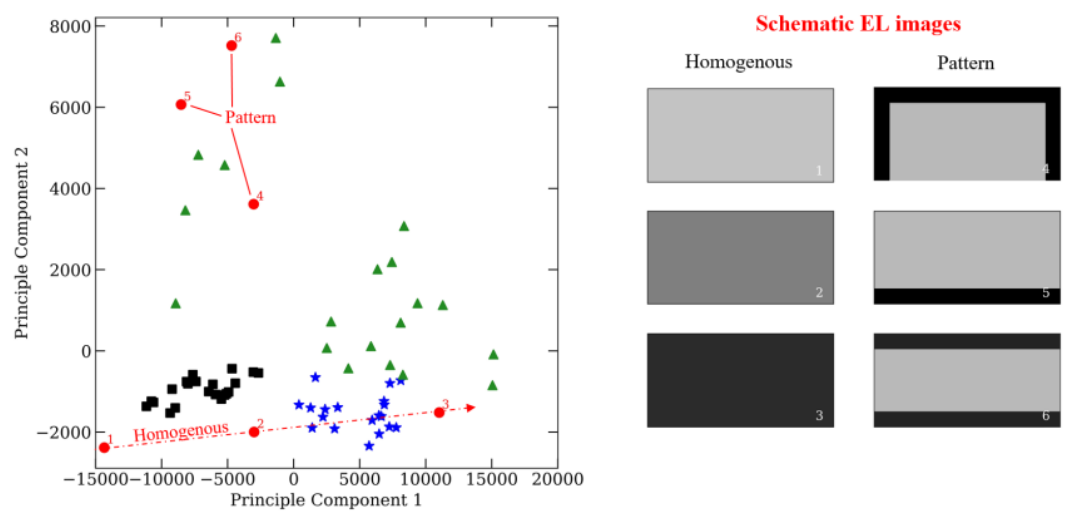

Fig. 5: Replot of PC relationships shown in Fig 4-c with additional results of schematic EL image shown on the right side. The schematic EL images represent a homogenous variation of the contrast and a specific pattern variation, as an example a pattern similar to those of the PID failure is chosen. 


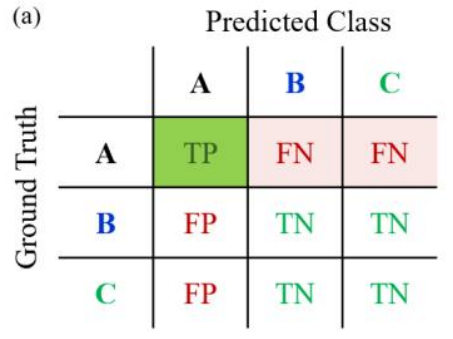

\begin{tabular}{l|c|c|c} 
(b) & \multicolumn{3}{|c}{ Predicted Class } \\
& A & B & C \\
\hline $\mathbf{A}$ & 6 & 0 & 0 \\
\hline $\mathbf{B}$ & 2 & 3 & 0 \\
\hline C & 0 & 1 & 6
\end{tabular}

(c) Example Test Images

Fig. 6: (a) General confusion matrix scheme for a three-class prediction, A=Reference, $B=$ LeTID, C=PID, (b) results of 18 test EL images used solely during kNN classification. (c) Examples of the prediction: true positive (TP) EL images of all classes, the false positive (FP) prediction of a ground truth LeTID image and the true negative prediction (TN) of a ground truth PID image as LeTID failure.
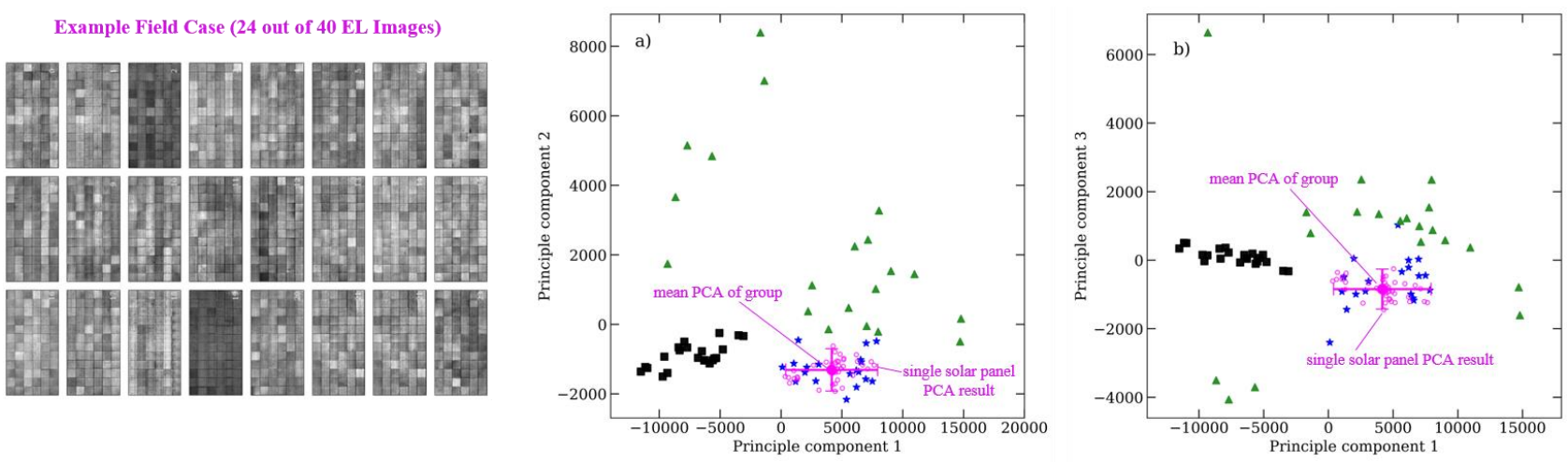

Fig. 7: Simulation of field case study using 40 EL images from field measurement. Results of PCA separation shown in fuchsia together with training data points already shown in Fig. 3. The mean value of the field case PCA is marked as a thicker symbol and used for the classification of the entire field data set. 